\title{
Twisting of Charged Nanoribbons to Helicoids Driven by Electrostatics
}

Gervasio Zaldivar, ${ }^{1}$ Martin Conda-Sheridan, ${ }^{2}$ and Mario Tagliazucchi. ${ }^{1,}{ }^{*}$

${ }^{1}$ Instituto de Química Física de los Materiales, Medio Ambiente y Energía and Departamento de Química Inorgánica Analítica y Química Física, University of Buenos Aires, School of Sciences, Ciudad Universitaria, Pabellón 2, Ciudad Autónoma de Buenos Aires C1428EHA, Argentina

2 Department of Pharmaceutical Sciences, College of Pharmacy, University of Nebraska Medical Center, Omaha, Nebraska 68198-6125, United States

* author to whom correspondence should be addressed.

E-mail:mario@qi.fcen.uba.ar 


\section{Numerical implementation}

We solved equations 2-5 of the main text using the finite-element method (FEM) implemented in COMSOL Multiphysics ${ }^{\circledR}$ 5.2. We built a rectangular calculation box containing the nanoribbon, which is described as a parametric surface (therefore, the ribbon has no thickness). Figure S1a shows a snapshot of the calculation box for the case of a helicoid-shaped ribbon. The parametrization of the surface of the helicoid is:

$$
\begin{aligned}
& x\left(s_{1}, s_{2}\right)=-\frac{w}{2} s_{1} \sin \left(\pi s_{2}\right) \\
& y\left(s_{1}, s_{2}\right)=-\frac{w}{2} s_{1} \cos \left(\pi s_{2}\right) \\
& z\left(s_{1}, s_{2}\right)=s_{2} P
\end{aligned}
$$

where $w$ is the width of the ribbon, $P$ is the period of twisting, $s_{1}$ is a parameter that takes values from -1 to 1 , and $s_{2}$ is a parameter that takes values from 0 to 1 . This parametrization defines a left-handed helicoid.

We used periodic boundary conditions at the back and front faces of the calculation box (colored in yellow in Fig. Figure S1a) and bulk boundary conditions at the lateral faces (colored blue in Fig. Figure S1a), i.e. we set the electrostatic potential to zero and the ion concentrations to their bulk values at these lateral faces.

In all calculations, the lateral size of the calculation box was set to be at least two orders of magnitude larger than the Debye length, $\lambda_{D}$. We used a triangular mesh that was finer near the nanoribbon surface than in the bulk. The maximum size of the elements of the grid near the surface was set to be $\sim 0.1 \lambda_{\mathrm{D}}$. The results are converged with respect to the sizes of the calculation box and the mesh. Results for left-handed and right-handed helicoid were exactly equivalent, as expected. Finally, the free-energy $\Omega^{*}$ for the helicoid with $P \rightarrow \infty$ converged to that of the flat ribbon, as expected (see Figure 2 in the main text).
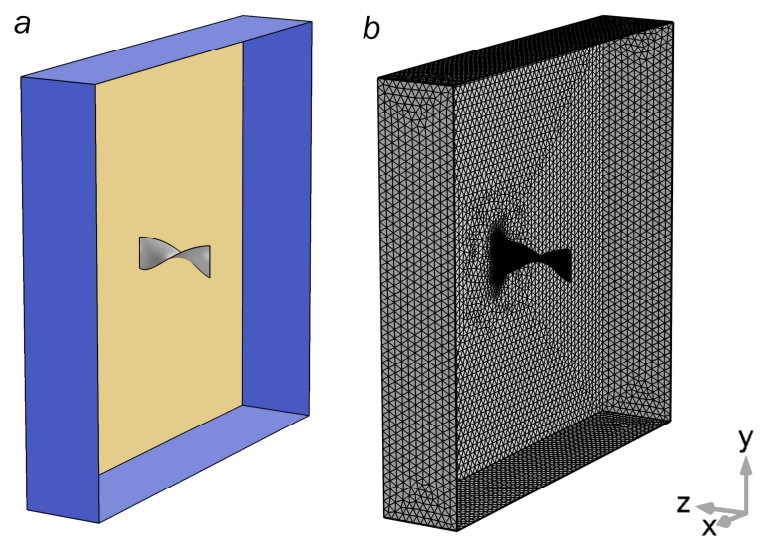

Figure S1. Snapshots of (a) a COMSOL calculation box containing a helicoid-shaped nanoribbon and (b) mesh used in the calculation. Bulk boundary conditions are imposed to the lateral faces (colored in blue in panel a) and periodic boundary conditions were used for the front (invisible) and back (colored in yellow) faces. 


\section{Constant-perimeter and constant-length twisting}

Figure S2 shows the excess free energy of twisting $\left(\Delta \Omega^{*}\right)$ as a function of the period for constant-length twisting (Figure S2a) and constant-perimeter twisting (Figure S2b), for a nanoribbon with $w=20 \mathrm{~nm}$ and a salt concentration of $0.005 \mathrm{M}$. The behavior of $\Delta \Omega^{*}$ is dominated by the effect of the transformation on the surface charge density. Constant-length twisting enlarges the area of the ribbon, therefore its surface charge density diminishes. This effect decreases electrostatic repulsions between surface charges and, thus, the helicoid becomes more stable than the flat ribbon. On the other hand, constant-perimeter twisting shrinks the area of the ribbon and enlarges the surface charge density. This effect increases the electrostatic repulsions and, as a consequence, the helicoid becomes less stable than the flat ribbon. Note that both constant-length and constant-perimeter transformations greatly modify the average distances between charges on the surface. In a self-assembled nanoribbon, the intermolecular distances are fixed by interactions at the molecular lengthscale; therefore, twisting of the ribbon shouldn't affect the surface charge density. Hence, the constant-area transformation (which keeps a fixed surface charge density) is the only physically meaningful twisting transformation.
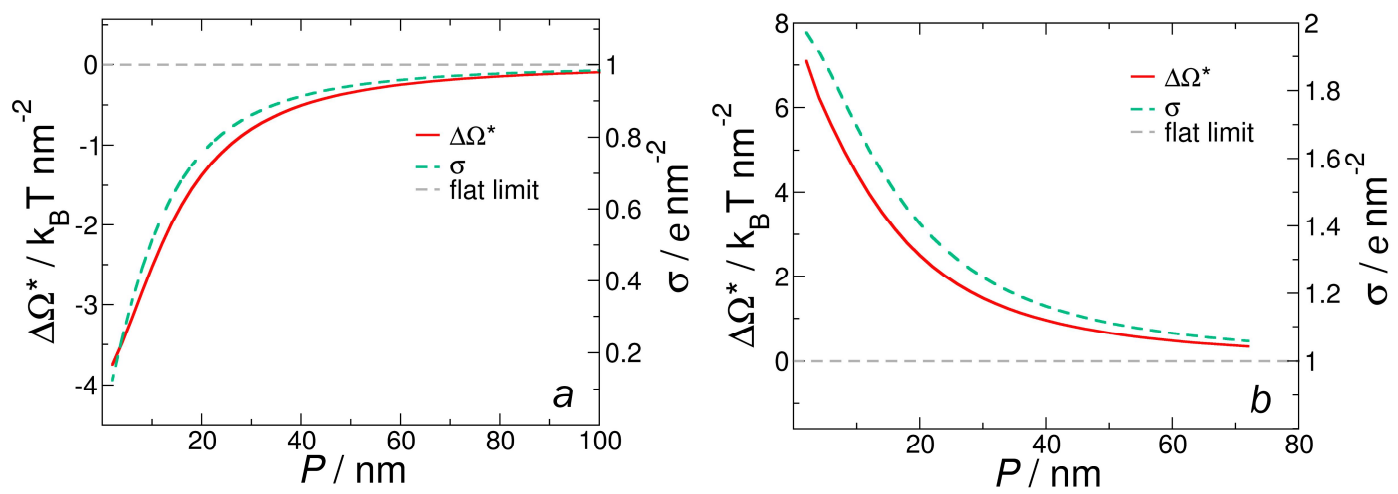

Figure S2. Surface charge density ( $\sigma$, green dashed lines, right axis) and planar ribbon-to-helicoid excess free-energy difference $\left(\Delta \Omega^{*}\right.$, red solid lines, left axis) as a function of the period of twisting $(P)$. The transformation was performed at constant ribbon length $(a)$ and at constant ribbon perimeter $(b)$. Salt concentration was set to $0.005 \mathrm{M}$ and the width of the ribbon to $20 \mathrm{~nm}$.

\section{Stability of ribbons of different shapes}

Figure S3 show the excess free energy $\left(\Delta \Omega^{*}\right)$ involved in the constant-area transformation of a flat ribbon into a longitudinally bent ribbon (Fig. S3a), a transversely bended ribbon (Fig. S3b) and a helical ribbon (Fig. S3c), see shapes in Figure 1 in the main text. For all these transformations, $\Delta \Omega^{*}$ is always positive, which indicates that the twisted or bent ribbons are always less stable than the flat ribbon. In all cases, $\Delta \Omega^{*}$ increases as the degree of deformation increases, i.e. as the shape of the nanoribbon departs from the flat one. 

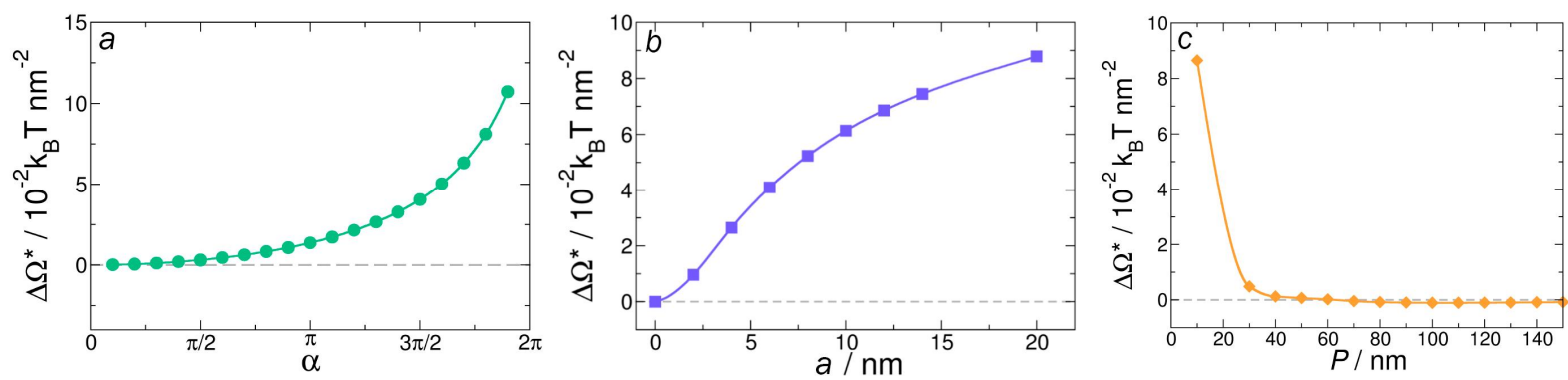

Figure S3. Excess free-energy difference $\left(\Delta \Omega^{*}\right)$ involved in the transformation of a flat ribbon into: $(a)$ a longitudinally bended ribbon, $(b)$ a transversally bended ribbon and $(c)$ a helical ribbon. The $\mathrm{x}$ axis corresponds to: the angle of bending (panel $a$ ), the amplitude of bending (panel $b$ ) and the period of helical twisting (panel $c$ ). The width of the ribbon is $20 \mathrm{~nm}$ and the salt concentration is $0.005 \mathrm{M}$. The radius of the helical ribbon in panel $c$ is $10 \mathrm{~nm}$.

\section{Perimeter-to-area ratio of a helicoid}

The area of a helicoid is given by:

$A_{\text {helicoid }}=N \pi\left[(P / \pi)^{2} \ln \left(\frac{w+\sqrt{(2 P / \pi)^{2}+w^{2}}}{2 P / \pi}\right)+w / 4 \sqrt{(2 P / \pi)^{2}+w^{2}}\right]$

where $N$ is the number of twists, and $P$ and $w$ are the period and the width of the helicoid, respectively.

For a very long helicoid (so that the contribution of the edges at both ends to the total perimeter is negligible), the perimeter is given by:

$p_{\text {helicoid }}=N \pi \sqrt{(2 P / \pi)^{2}+w^{2}}$

Therefore, the perimeter-to-area ratio is:

$$
r_{\text {helicoid }}=\frac{\sqrt{(2 P / \pi)^{2}+w^{2}}}{(P / \pi)^{2} \ln \left(\frac{w+\sqrt{(2 P / \pi)^{2}+w^{2}}}{2 P / \pi}\right)+w / 4 \sqrt{(2 P / \pi)^{2}+w^{2}}}
$$

The perimeter and area of a very long flat ribbon are given by:

$A_{\text {flat }}=w L$

and

$p_{\text {flat }}=2 L$

respectively. In these expressions, $L$ and $w$ are the length and the width of the ribbon, respectively. Therefore, the perimeter-to-area ratio of the flat ribbon is:

$r_{\text {flat }}=2 / w$

Dividing eq. S4 by eq. S7 yields to the perimeter-to-area ratio of a helicoid relative to that of a flat ribbon, given by: 


$$
\frac{r_{\text {helicoid }}}{r_{\text {flat }}}=\frac{w / 2 \sqrt{(2 P / \pi)^{2}+w^{2}}}{(P / \pi)^{2} \ln \left(\frac{w+\sqrt{(2 P / \pi)^{2}+w^{2}}}{2 P / \pi}\right)+w / 4 \sqrt{(2 P / \pi)^{2}+w^{2}}}
$$

Rearranging equation S8, one obtains:

$$
\frac{r_{\text {helicoid }}}{r_{\text {flat }}}=\frac{2 \sqrt{(2 P / w \pi)^{2}+1}}{(2 P / w \pi)^{2} \ln \left(\frac{1+\sqrt{(2 P / w \pi)^{2}+1}}{2 P / w \pi}\right)+\sqrt{(2 P / \pi w)^{2}+1}}
$$

Equation S9 shows that $r_{\text {helicoid }} / r_{\text {flat }}$ only depends on the dimensionless parameter $P / w$.

\section{Analysis of thermal fluctuations.}

Let us consider the ribbon as composed by a sequence of rows of molecules, see Figure S4a, where each cylinder in the Figure represents a row of molecules. Each row has a rotation angle $\theta$ with respect to the following one, a thickness in the direction of the ribbon of $a$ (which is equal to the distance between molecules) and a width of $w$ in the normal direction (which is equal to the width of the ribbon). In the present case, $a=\sigma^{-1 / 2}=1 \mathrm{~nm}$. We will assume that the rows always rotate in the same direction $(\theta>0)$. We made this choice based on the experimental fact that twisting generally happens in systems with chiral interactions that guide the rotation towards a specific direction. This analysis cannot be applied to purely electrostatic twisting, where the rows could indistinctly rotate to the right or to the left. For such cases, a more complex analysis of the thermal fluctuations is required, which is out of the scope of this work. 

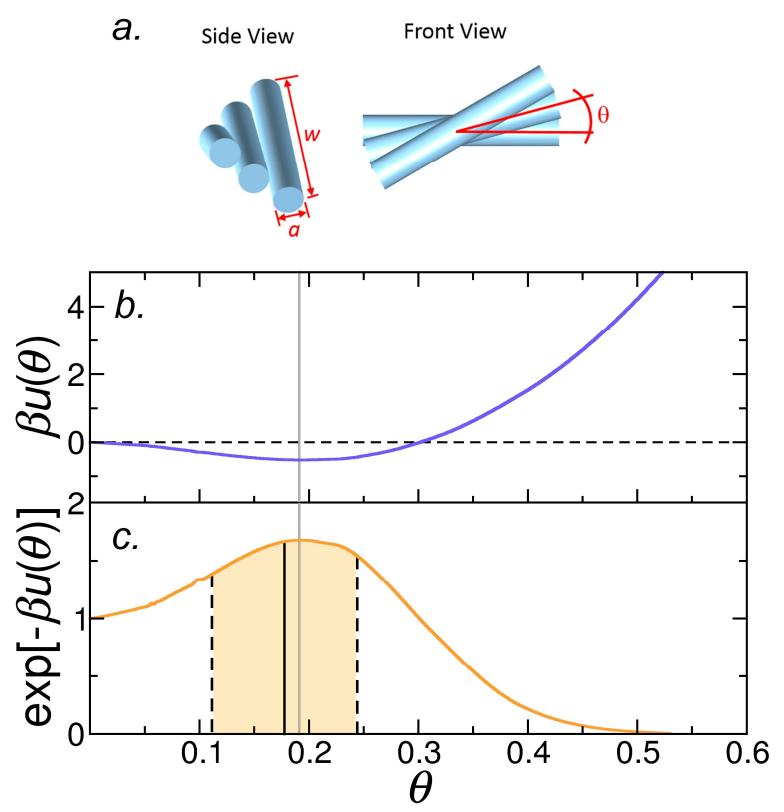

Figure S4. a. Schematic representation of the sequence of rows of molecules forming the ribbon. b. Energy of rotating two adjacent rows, $\beta u(\theta)$, as a function of the rotation angle (in radians). c. Boltzmann factor, $\exp [-\beta u(\theta)]$, as a function of $\theta$. The vertical gray line in panels $b$ and $c$ indicates the most stable rotation angle neglecting thermal fluctuations, which was obtained from the plot in Figure $2 a$ in the main text. The black solid line and the black dashed lines in panel $c$ indicate the average rotation angle, $\langle\theta\rangle$, and its upper and lower boundaries, $\langle\theta\rangle \pm 3\left\langle\delta \theta^{2}\right\rangle^{1 / 2}$, respectively. In this model we assume that the rotation occurs always in the same direction $(\theta>0)$ and, therefore, assumes the presence of molecular chirality that favors rotation in that direction.

The energy required to rotate two adjacent rows by the angle $\theta$ can be obtained from $\Delta \Omega$ vs $P$ plots (see Figure $2 \mathrm{a}$ in the main text). The rotation angle for a given period $P$ is

$\theta=\frac{\pi}{P} a$

and the energy is

$u(\theta)=\Delta \Omega \delta A$

where $\Delta \Omega$ is the excess free energy per unit area (units of $k_{\mathrm{B}} T / \mathrm{nm}^{2}$ ) and $\delta A$ is the area of the row of molecules. Figure S4b shows a plot of $\Delta \Omega(\theta)$ for the results in Figure $2 \mathrm{a}$ in the main text corresponding to a salt concentration of $0.005 \mathrm{M}$.

Let us consider a ribbon with a length equal to $L$. This ribbon has $N=L / a$ rows of molecules. The rotation of each row is assumed to be independent of the other ones, therefore the total partition function for the system is:

$Q=q^{N-1}$

where $q$ is the partition function for a single rotation between two adjacent rows (note that in a system of $N$ rows, there are $N-1$ rotations), given by:

$q=\int_{0}^{\theta_{\max }} \exp (-\beta u(\theta)) d \theta$ 
In this integral $\theta^{\max }$ is a cut-off value for which the integrant becomes very small. The average rotation of the whole ribbon, $\langle\Theta\rangle$, is:

$\langle\Theta\rangle=(N-1)\langle\theta\rangle$

where

$\langle\theta\rangle=\frac{1}{q} \int_{0}^{\theta_{\max }} \theta \exp (-\beta u(\theta)) d \theta$

Its variance is:

$$
\left\langle\delta \Theta^{2}\right\rangle=(N-1)\left\langle\delta \theta^{2}\right\rangle=(N-1)\left(\left\langle\theta^{2}\right\rangle-\langle\theta\rangle^{2}\right)
$$

where

$$
\left\langle\theta^{2}\right\rangle=\frac{1}{q} \int_{0}^{\theta_{\max }} \theta^{2} \exp (-\beta u(\theta)) d \theta
$$

Figure S4c shows the Boltzmann factor, $\exp [-\beta u(\theta)]$, as a function of $\theta$. Using the data in Figure S4c, we obtain $\langle\theta\rangle=0.18 \mathrm{rad}$ and $\left\langle\delta \theta^{2}\right\rangle^{1 / 2}=0.10 \mathrm{rad}$. We define lower and upper boundaries for the rotation angle $\theta$ using three standard deviations: $\langle\theta\rangle \pm 3\left\langle\delta \theta^{2}\right\rangle^{1 / 2}$ (marked with dashed lines in Figure S4c). As expected, the equilibrium value of $\langle\theta\rangle$ (gray solid line in Figure S4b and Figure S4c), which includes thermal fluctuations, slightly differs from the most stable angle in the absence of fluctuations (black solid line).

Now we are interested in estimating the period of the ribbon. For a given ribbon, we will consider a single period, which corresponds to a full $180^{\circ}$ rotation (total rotation angle $\Theta=\pi$ ). Therefore, the period $\bar{P}$ (period including thermal fluctuations) is the length of the ribbon for which $\langle\Theta\rangle=(N-1)\langle\theta\rangle=\pi$ :

$$
\bar{P}=N a=\left(\frac{\pi}{\langle\theta\rangle}+1\right) a
$$

Accordingly, we define the upper and lower boundaries of $\bar{P}$ by considering three standard deviations of $\langle\Theta\rangle$. We first find the values of $N^{\text {upper }}$ and $N^{\text {lower }}$ that fulfill the conditions:

$$
\langle\Theta\rangle+3\left\langle\delta \Theta^{2}\right\rangle^{1 / 2}=\left(N^{\text {upper }}-1\right)\langle\theta\rangle+3 \sqrt{\left(N^{\text {upper }}-1\right)\left\langle\delta \theta^{2}\right\rangle}=\pi
$$

and

$$
\langle\Theta\rangle-3\left\langle\delta \Theta^{2}\right\rangle^{1 / 2}=\left(N^{\text {lower }}-1\right)\langle\theta\rangle-3 \sqrt{\left(N^{\text {lower }}-1\right)\left\langle\delta \theta^{2}\right\rangle}=\pi
$$

and then define the upper and lower boundaries of $\bar{P}$ as:

$\bar{P}^{\text {upper }}=N^{\text {upper }} a$

and 
$\bar{P}^{\text {lower }}=N^{\text {lower }}$ a

respectively.

Equations S18, S21 and S22 yield $\bar{P}=15.9 \mathrm{~nm}, \bar{P}^{\text {lower }}=10.0 \mathrm{~nm}$ and $\bar{P}^{\text {upper }}=25.7 \mathrm{~nm}$.

We stress again that our model assumed twisting in only one direction $(\theta>0)$ and, therefore, it is only applicable in the presence of chiral interactions breaking the symmetry. In the case of purely electrostatic interactions, right-handed and left-handed rotations occur with equal probability and, therefore, $\langle\theta\rangle=0$. In that case, twisting will be observed if subsequent rotations are correlated (this correlation was not included in the simple model above). In other words, if the correlation between one rotation and the next is strong enough, the size of the twisted domains will be larger than the equilibrium twisting period and short-range order (i.e. in the lengthscale of a few periods) will be observed in the ribbon, although long-range order will be lost.

\section{Comparison between numerical and analytical Poisson-Boltzmann (PB) calculation and Yukawa approximation for the free-energy of opposing charged surfaces.}

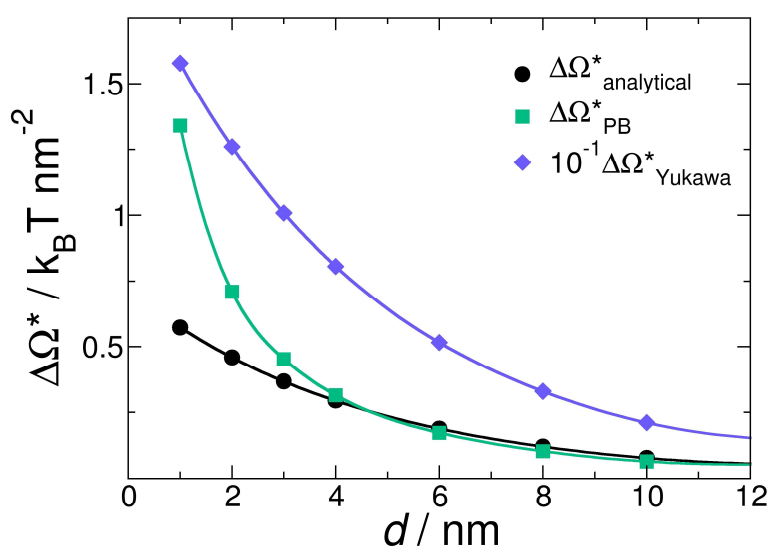

Figure S5. Excess free-energy difference of two parallel, infinite planes bearing a constant surface charge density as a function of the separation between plates, $d$. The calculations correspond to numerical calculations with the Poisson-Boltzmann model (green squares), the Yukawa model (blue diamonds) and an approximated analytical expression of the Poisson-Boltzmann model (black circles, see Ref. 1). The surface charge density of the planes was set to $1|e| \mathrm{nm}^{-2}$. Salt concentration: $0.005 \mathrm{M}$.

In order to compare the predictions of the nonlinear PB model and the Yukawa pairwise additive model described in the main text, we calculate the excess free-energy difference, $\Delta \Omega^{*}$, of two parallel infinite planes with a constant surface charge density as a function of the distance between them $(d)$, using the numerical Poisson-Boltzmann model, an approximate analytical solution of Poisson-Boltzmann equation (see ref. [1]) and the Yukawa model (eq. 6 in the main text), see Figure S5. The approximate analytical PB model compares very well with the numerical results for large distances and starts to fail when the planes approach. On the other hand, even though the Yukawa model predicts reasonably well the qualitative behavior of $\Delta \Omega^{*}$ vs $d$, the predicted values of $\Delta \Omega^{*}$ are more than one order of magnitude larger than those predicted by 
the PB model for the whole range of $d$ (note that the $\Delta \Omega^{*}$ values for the blue line in Figure S4 has been divided by a factor of 10). This result is in agreement with the predictions of $\Delta \Omega^{*}$ for the twisting of a flat ribbon to a helicoid presented in Figure 4 in the main text.

\section{References}

[1] Israelachvili, J. N. Intermolecular and Surface Forces, 3rd ed.; Academic Press: Burlington, MA, 2011; pp 317-318. 